\title{
p53 gene product expression in resected non-small cell carcinoma of the lung, with studies of concurrent cytological preparations and microwave antigen retrieval
}

\author{
S Binks, C A Clelland, J Ronan, J Bell
}

\begin{abstract}
Aim-To document the frequency and extent of p53 gene product expression in paraffin sections of resected non-small cell carcinoma of the lung and in cytological preparations of the same tumours; to determine the effect of microwave antigen retrieval on antigen detection.
\end{abstract}

Methods-Representative paraffin sections of 50 non-small cell carcinomas were stained with an antibody to p53 gene product (DO-7) both with and without prior microwave antigen retrieval. Cytoblocks and cell smears obtained from 19 cases were similarly stained.

Results-Using a histochemical scoring system (0-300) which takes into account staining intensity and extent, $78 \%(n=39)$ of microwave pretreated paraffin sections and $52 \%(n=26)$ of non-pretreated sections scored between 5 and $300 ; p=0.001$; $56 \%(n=28)$ of microwave pretreated sections and only $2 \% \quad(n=1)$ of nonpretreated sections scored between 100 and $300(p=0.0001) ; 75 \%$ of direct smears of tumours and $80 \%$ of cytoblocks stained similarly to the paraffin sections of the resected specimens. No smears or cytoblocks stained positively when the sections of the resected specimen were negative.

Conclusions-As up to $78 \%$ of non-small cell lung carcinomas overexpress p53 gene product, this may prove to be a valuable diagnostic method in biopsy or cytological material when the morphological diagnosis is uncertain. Microwave antigen retrieval is effective on formalin fixed tissue. (F Clin Pathol 1997;50:320 323)

Keywords: lung neoplasm; immunocytochemistry; p53

Department of Histopathology, City Hospital, Nottingham S Binks

C A Clelland

J Ronan

J Bell

Correspondence to: Dr C A Clelland, Histopathology, City Hospital, Hucknall Road, Nottingham NG5 1 PB.

Accepted for publication 22 January 1997
There has been much interest in p53 gene mutation and upregulation in lung cancer. ${ }^{13} \mathrm{~A}$ convenient way of identifying an abnormality in the p53 gene is to stain the gene product immunohistochemically, enabling wild-type p53 gene product in excess and the more stable mutated forms of $\mathrm{p} 53$ gene product to be visualised. ' Increasing the sensitivity of this test can be achieved by microwave antigen retrieval on paraffin wax sections." As previous reports have reliably recorded that most non-small cell lung carcinomas (NSCLC) overexpress p53 gene product (p53GP), ${ }^{7}$ we wished to document the extent and intensity of p53GP using optimum staining conditions, ${ }^{11}$ including microwave antigen retrieval. We therefore stained paraffin sections of 50 resected NSCLC. If a large proportion of NSCLC stain positively for p53GP, then under certain circumstances - such as a small bronchial biopsy or cytological preparation-the stain may have a role as a diagnostic test of malignancy. Therefore we decided also to stain cytological preparations including direct smears and cytoblock samples in order to test the reliability of staining on samples other than paraffin sections of resected tissue, particularly samples where diagnostic difficulty may be encountered.

\section{Methods}

Fifty cases of NSCLC were retrieved from the files of the Department of Histopathology, City Hospital, Nottingham. All the cases were resection specimens for attempted primary surgical cure. The diagnoses were reviewed and cases subtyped accordingly. There were 32 squamous cell carcinomas, 15 adenocarcinomas, and three adenosquamous carcinomas. In this department every effort is made to fully categorise NSCLC, and the category of large cell carcinoma is therefore rarely used-none being included in this study. Dewaxed paraffin sections on "TESPA" coated slides immersed in $0.1 \mathrm{M}$ sodium citrate buffer at $\mathrm{pH} 6.0$ were pretreated by boiling for 15 minutes at full power in an $800 \mathrm{~W}$ microwave oven. Identical slides were processed without microwave antigen retrieval. DO-7 antibody (an anti-p53 gene product antibody produced by Novocastra (Newcastle, UK) with reactivity for human wild-type $\mathrm{p} 53$ and mutant $\mathrm{p} 53$ proteins (amino acids $1-45$ )) was applied at dilution of 1 in 50 for 45 minutes, following preliminary dilution experiments - as the manufacturer's recommended dilution of 1 in 100 to 1 in 200 was found to be unsatisfactory. A standard avidinbiotin complex method was used, with diaminobenzidine applied for 10 minutes. Slides were weakly counterstained in haematoxylin.

Cytological preparations were made from 19 resected specimens (16 smears and 15 cytoblocks) by passing a screw needle ( $24 \mathrm{G}$, Meditech, Massachusetts, USA) twice into the tumour to make direct smears and twice into the tumour for preparation of a cell suspension in formalin. Direct smears were fixed immedi- 


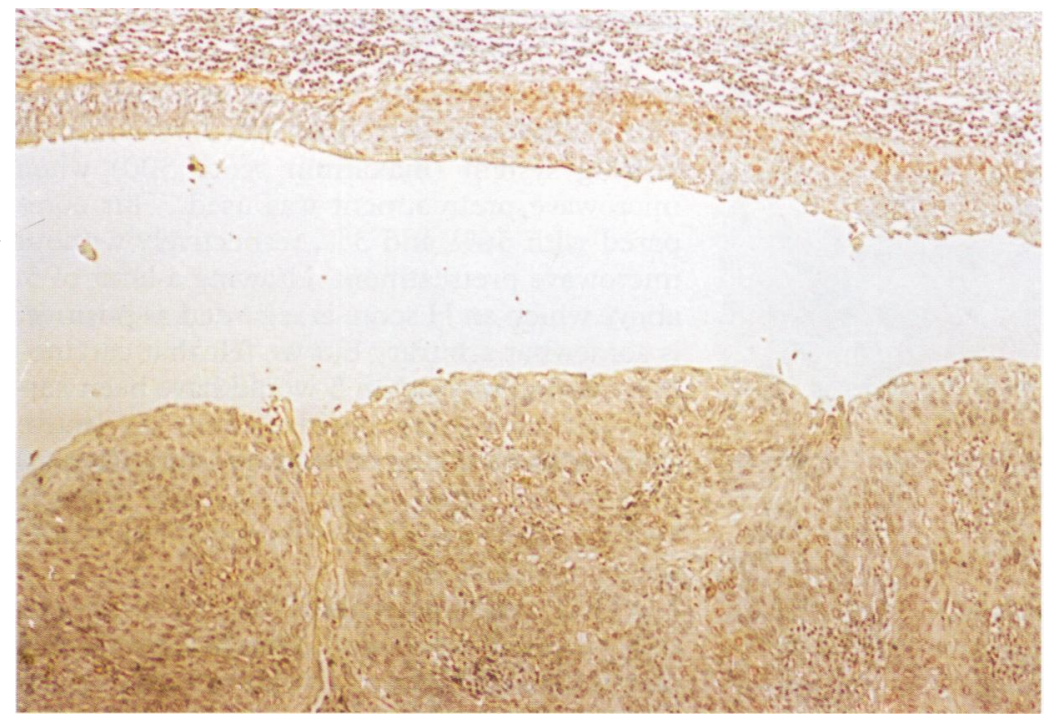

Figure 1 Case 47: no microwave enhancement, $H$ score 49.5. Weak, patchy positivity is seen.

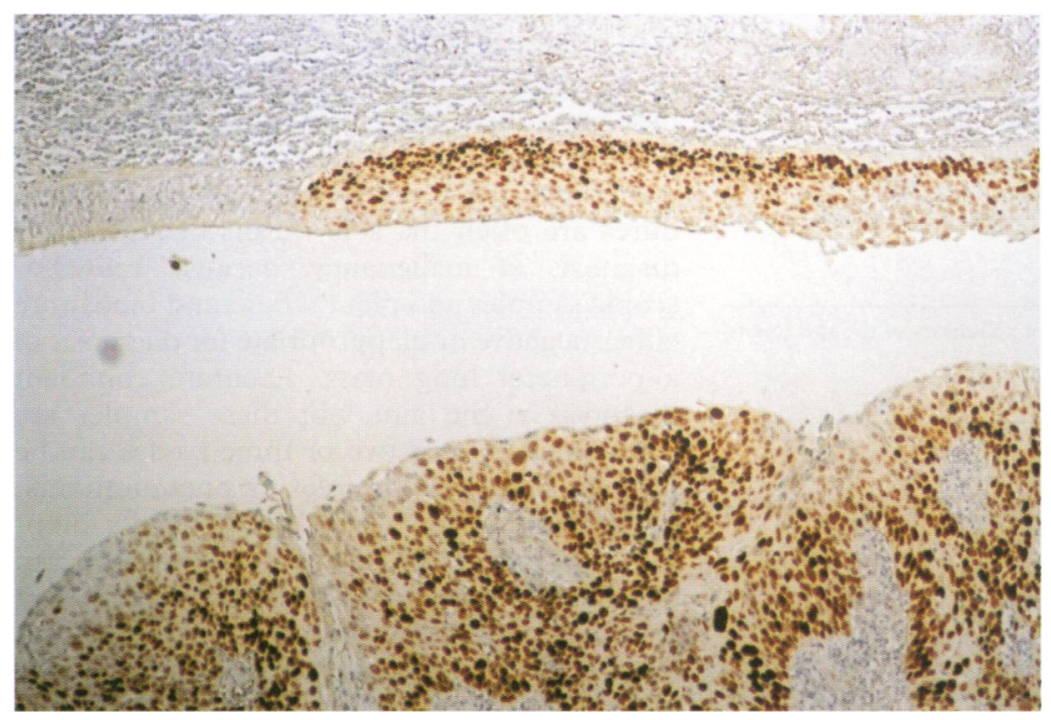

Figure 2 Case 47: with microwave enhancement, $H$ score 156. Strong positivity of the in situ component, mainly the basal part of the epithelium, is present in addition to the invasive squamous carcinoma.

ately in acetic alcohol and stained by Papanicolou's method. Cytoblocks were made according to the manufacturer's protocol (Shandon, Pittsburgh, USA). Immunostaining of the smears and sections of cytoblocks was as above except that microwave antigen retrieval was not required for the smears because they were fixed in acetic alcohol rather than formalin.

The immunostaining of paraffin sections of the resected carcinomas was analysed using a histochemical scoring system ( $\mathrm{H}$ score). ${ }^{12}$ Twenty random microscope fields per slide viewed at $\times 200$ magnification were scored. The percentage of tumour nuclei in each field corresponding to the following staining intensi-

Table 1 The frequency of $H$ scores with microwave enhancement for each histological type Table 1 The frequency of $H$ scores with microwave enhancement for each histological type
of lung cancer is shown. Numbers of cases without microwave enhancement in parentheses

\begin{tabular}{lllll}
\hline & $<5$ & $5-100$ & $100-200$ & $200-300$ \\
\hline All $(\mathrm{n}=50)$ & $11(24)$ & $11(25)$ & $21(1)$ & 7 \\
Squamous carcinoma $(\mathrm{n}=32)$ & $6(14)$ & $6(17)$ & $16(1)$ & 4 \\
Adenocarcinoma $(\mathrm{n}=15)$ & $4(9)$ & $5(6)$ & 4 & 2 \\
Adenosquamous carcinoma $(\mathrm{n}=3)$ & $1(1)$ & $0(2)$ & 1 & 1 \\
\hline
\end{tabular}

ties was estimated: negative $(0)$, weakly positive (1), moderately positive (2), or strongly positive (3). A score between 0 and 300 was obtained. For example:

Case 4: 4th field

\begin{tabular}{lll}
\hline Intensity & Percentage of nuclei & Weighted \\
\hline 0 & 0 & 0 \\
1 & 20 & 20 \\
2 & 20 & 40 \\
3 & 60 & 180 \\
\hline
\end{tabular}

Total $\mathrm{H}$ score $=240$

Smears and cytoblocks were recorded as positive, negative, or inadequate when there was no assessable material.

\section{Results}

RESECTION SPECIMENS

The majority of NSCLC scored more than 5 on a histochemical scoring system when stained for p53GP, whether or not microwave antigen retrieval was used (78\% with $v 52 \%$ without) but the difference (figs 1 and 2) was statistically significant ( $p=0.001$; MannWhitney U test) (table 1). All histological subtypes of NSCLC showed this trend with $81 \%$ of squamous carcinomas, $73 \%$ of adenocarcinomas, and two of three adenosquamous carcinomas scoring more than 5 , the respective percentages without microwave pretreatment being $56 \%, 40 \%$, and $67 \%$. Not only did weakly staining tumours become negative (fig 3) when microwave treatment was omitted, but high $\mathrm{H}$ scores were rare, only one case scoring over 100 compared with 28 cases when pretreatment was applied.

Qualitatively, the staining of tumour nuclei was patchy in distribution and variable in intensity: the cells at the edge of islands of tumour were often more strongly positive than those in the centre. Dysplastic bronchial epithelium adjacent to squamous carcinoma was also often DO-7 positive (fig 2), but strong staining of morphologically normal bronchial epithelium and other cell types was not observed. However, there was weak positive staining of occasional epithelial and stromal cell nuclei in some cases where microwave pretreatment was applied. Also in these cases there was variable staining of collagen and cartilage.

\section{CYTOLOGICAL PREPARATIONS}

Five of 15 cytoblocks and one of 16 direct smears were inadequate. Two cytoblocks and three smears were p53GP negative when the resected specimen was positive but in all other cases staining was as for the resected specimen (table 2). Of the nine cases with p53GP positive smears, seven of the resected tumours had $\mathrm{H}$ scores greater than 100 with antigen retrieval. The $\mathrm{H}$ scores of the three p53GP positive cases with negative smears were 85,97 , and 243. Staining of structures other than tumour nuclei was not observed in the cytological preparations.

\section{Discussion}

p53GP overexpression is common in human malignancies and particularly common in nonsmall cell lung cancer. While there are difficulties in interpreting the biological significance of 


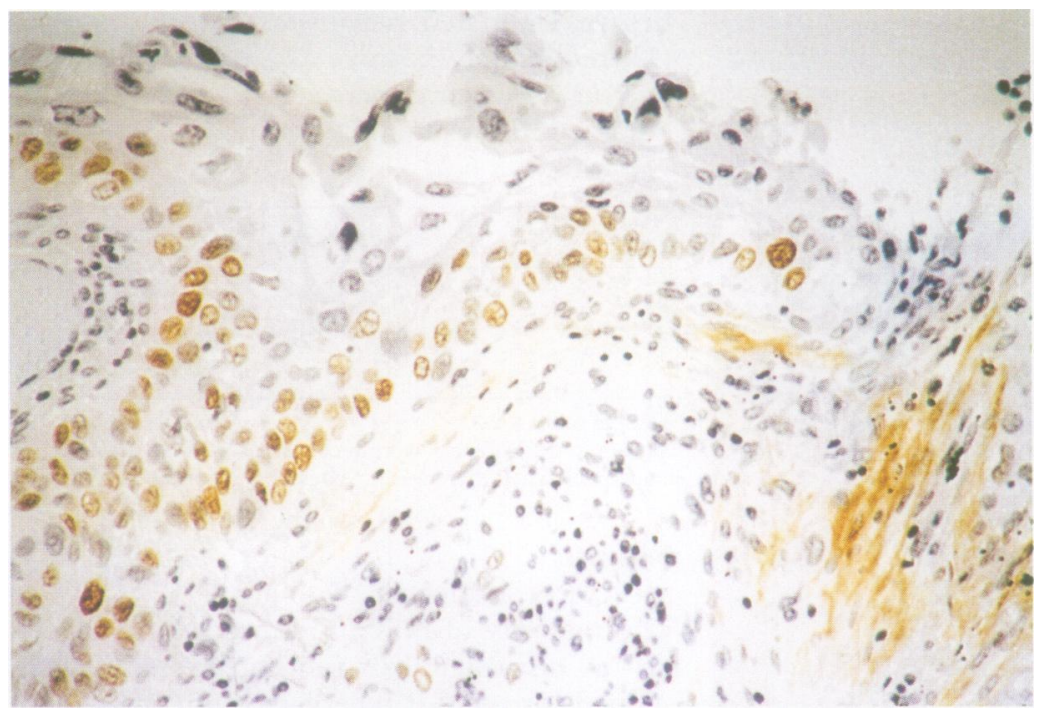

Figure 3 Case 22: without microwave enhancement, $H$ score 0.5 ; with, $H$ score 73 . This squamous carcinoma was negative but in the microwaved section in this figure some of the surface epithelium is positive. Non-specific staining of collagen is also seen.

Table 2 p53GP immunostaining in paraffin sections of resected lung cancers compared with staining of cytological preparations

\begin{tabular}{lllllll}
\hline & \multicolumn{2}{c}{ Smears } & & & \multicolumn{2}{l}{ Cytoblocks } \\
\cline { 2 - 3 } & + & - & & + & - \\
\hline Resected & + & 9 & 3 & & 4 & 2 \\
Tumour block & - & 0 & 3 & & 0 & 4
\end{tabular}

One smear and six cytoblocks were inadequate because of very poor cellularity of the specimens.

Table 3 p53 immunostaining in previous reports

\begin{tabular}{|c|c|c|c|c|}
\hline Reference & $n$ & $\begin{array}{l}\% \text { p53GP } \\
\text { positive NSCLC }\end{array}$ & $\begin{array}{l}\text { Microwave } \\
\text { enhancement }\end{array}$ & Material \\
\hline Caamano et a $\mathcal{l}^{\beta}$ & 50 & 46 & None & Fresh \\
\hline Porter et al $^{14}$ & 20 & 55 & None & Fresh \\
\hline McLaren et al 9 & 20 & 56 & None & Fresh \\
\hline Iggo et al & 31 & 65 & None & Fresh \\
\hline Vahakangas et $a l^{15}$ & 11 & 45 & None & Fixed \\
\hline Quinlan et $a l^{16}$ & 114 & 43 & None & Fixed \\
\hline Soini et $a l^{17}$ & 27 & 63 & None & Fixed \\
\hline Korokopoulou et $a l^{8}$ & 27 & 44 & None & Fixed \\
\hline Westra et $a l^{10}$ & 70 & 47 & None & Fixed \\
\hline Ebina et $a l^{18}$ & 123 & 39 & None & Fixed \\
\hline Dalquen $e t a l^{19}$ & 247 & 48 & None & Fixed \\
\hline
\end{tabular}

NSCLC $=$ non-small cell lung carcinoma.

p53GP positivity demonstrated by immunohistochemistry when the molecular abnormalities of the p53 gene are not known, immunopositivity is significant diagnostically. This is true because normal bronchial epithelium and stroma are generally negative or may show focal, weak staining..$^{13}$ Immunopositivity occurs early in the process of oncogenesis and this is reflected by the frequent staining of dysplastic bronchial epithelium. ${ }^{7}$ Strong staining is therefore a good marker of bronchial dysplasia and malignancy.

In previous studies, the frequency of $\mathrm{p} 53 \mathrm{GP}$ positivity in NSCLC ranged from $39-65 \%$ (table 3). In most studies, whether using fresh, frozen, or formalin fixed tissue without microwave antigen retrieval, about half of the cases were found to be positive. However, with microwave antigen retrieval, as in this study, this proportion increases to $78 \%$. There may be some variations in the immunohistochemical technique and indeed in the antibodies used, but in our controlled study microwave antigen retrieval significantly increased the rate of detection of p53GP. Eighty one per cent of squamous carcinomas scored more than 5 and $62.5 \%$ scored over 100 on our histochemical scoring system (maximum score 300) when microwave pretreatment was used. This compared with $56 \%$ and $3 \%$, respectively without microwave pretreatment. Drawing a limit of 5, above which an $\mathrm{H}$ score is regarded as positive, is somewhat arbitrary but we felt that carcinomas scoring more than 5 would have been categorised as weakly positive on a subjective scale. Also by taking an average score from 20 microscopic fields, cases with focal positive staining ( $\mathrm{H}$ score $>100$ in a single field) would score more than 5 .

p53GP immunostaining has been successfully applied to many different cytological preparations. ${ }^{20}$ However, we know of no reports of its use in screw needle cytology of lung cancers, although we have compared the use of the technique in both pleural cytology and biopsy in the diagnosis of malignant pleural effusion. ${ }^{21}$ Recently, studies have even succeeded in identifying p53 gene mutations in cytological samples from lung cancers using the polymerase chain reaction. ${ }^{22}$

Samples from screw needle biopsy procedures are often the sole means of reaching a diagnosis of malignancy, because bronchoscopic samples and pleural fluid and biopsy are either negative or inappropriate for diagnosis of a peripheral lung mass. Accurate confident diagnosis is the aim, but these samples are often small as only two or three passes can be made without serious risk of a pneumothorax.

Cytological samples of screw needle biopsies, whether direct smears or cytoblocks, can be stained immunocytochemically and in most cases the staining is consistent with the immunoreactivity of the resected specimen. When the cytological sample is small it is possible that a positive tumour with a low $\mathrm{H}$ score will not be recognised on cytology. Alternatively, vigorous antigen retrieval in paraffin sections of a resection specimen may be another reason for a weakly positive tumour with negative staining on cytology; acetic alcohol fixed smears, however, should not require unmasking of antigens. We avoided the use of formol saline, acetone, and methanol as fixatives for cytological samples as their use gives inferior results. ${ }^{11}$ In our small series, three direct smears were negative when the resected tumour was positive. In two cases the $\mathrm{H}$ score was below 100 but in the third instance the $\mathrm{H}$ score was very high. In other respects the results support the hypotheses above: positive resected tumours with positive cytology had $\mathrm{H}$ scores of over 100 in seven of nine cases. We found that more cellular preparations were achieved by using direct smears as opposed to cytoblocks, although it is clear that fewer stains could be performed on direct smears. As a routine we use direct smears because of the high rate of inadequacy we have experienced with cytoblocks.

In summary, under certain circumstances where there is diagnostic difficulty in determining whether a biopsy or cytological sample of a 
lung lesion is benign or malignant, p53 immunoreactivity may be of value. If strongly positive, a diagnosis of malignancy is supported but if negative the stain is non-contributory. Care should be taken when interpreting low levels of staining, particularly if focal and if antigen retrieval has been used.

We thank Ms P Mitchell for typing the manuscript, Michael Coates and Pat Gadsdon for preliminary technical work, and $\mathrm{Mr}$ Woates and Pat Gadsdon for preliminary technical work, and Mr
W E Morgan, Mr F D Salama, and Mr F D Beggs for their cooperation in providing surgical resections.

1 Iggo R, Gatter K, Bartek J, Lane DP, Harris AL. Increased ggo R, Gatter K, Bartek J, Lane DP, Harris AL. Increased
expression of mutant forms of p53 oncogene in primary expression of mutant forms of p53

2 Chiba I, Takahashi T, Nau MN, D'Amico D, Curiel DT, Mitsudomi T, et al. Mutations in the p53 gene are frequent in primary, resected non-small cell lung cancer. Oncogene 1990;5:1603-10.

3 Caamano J, Rugeri B, Momiki S, Sickler A, Zhang SY, Klien-Szanto AJP. Detection of p53 in primary lung tumours and non-small cell carcinoma cell lines. $\mathrm{Am} \mathscr{J}$ Pathol 1991;139:839-45.

4 Wynford-Thomas D. p53 in tumour pathology: can we trust immunohistochemistry? f Pathol 1992;166:329-30.

5 Hall PA, Lane DP. p53 in tumour pathology: can we trust immunohistochemistry? - revisited! f Pathol 1994;172:1-4.

6 Cattoretti G, Pileri S, Parravicini C, Becker MHG, Poggi S, Bifulco C, et al. Antigen unmasking on formalin-fixed, paraffin-embedded tissue sections. $\mathcal{F}$ Pathol 1993;171:83para.

7 Fontanini G, Bignati S, Bigini D, Merlo GR, Ribecchini A, Angeletti CA, et al. Human non-small cell lung cancer. p53 protein accumulation is an early event and persists during metastatic progression. F Pathol 1994;1 74:23-31.

8 Korkolopoulou P, Oates J, Crocker J, Edwards C. p53 expression in oat and non-oat small cell lung carcinomas: correlations with proliferative cell nuclear antigen. $\mathcal{f}$ Clin Pathol 1993;46:1093-6.

9 McLaren R, Kuzu I, Dunnill M, Harris A, Lane DP, Gatter KC. The relationship of p 53 immunostaining to survival in carcinoma of the lung. Br 7 Cancer 1992;66:735-8.

10 Westra WH, Offerhaus, J Goodman S, Slebos RJC, Polak M Baas IO, et al. Overexpression of p53 tumour suppressor gene product in primary lung adenocarcinoma is associated with cigarette smoking. Am ₹ Surg Pathol 1993;17:213-20.

11 Dowell SP, Lane DP, Hall PA. The immunocytochemical detection of p53 protein in cytological preparations: technical considerations. Cytopathology 1994;5:76-81.

12 McCarty KS, Miller LS, Cox EB, Konrath J, McCarty KS Oestrogen receptor analysis. Arch Path Lab Med 1985;109: 716-21.

13 Walker C, Robertson LJ, Myskow MW, Pendleton N, Dixon GR. p53 expression in normal and dysplastic bronchial epithelium and in lung carcinomas. Br $\mathcal{F}$ Cancer 1994;70 297-303.

14 Porter PL, Gown AM, Kramp SG, Coltera MD. Widespread p53 overexpression in human malignant tumours. Am $\mathcal{F}$ Pathol 1992;140:145-53.

15 Vahakangas KH, Samet JM, Metcalf RA, Welsh JA, Bennett WP, Lane DP, et al. Mutations of p53 and ras genes in radon-associated lung cancer from uranium miners. Lancet 1992;339:576-80.

16 Quinlan DC, Davidson AG, Summers CL, Warden HE, Doshi HM. Accumulation of $\mathrm{p} 53$ protein correlates with a poorer prognosis in human lung cancer. Cancer Res 1992;52:4828-31.

17 Soini Y, Kamel D, Nuorva K, Lane DP, Dahakangas K, Paako P. Low p53 protein expression in salivary gland tumours compared with lung carcinomas. Virchows Arch 1992;421:415-20.

18 Ebina $M$, Steinberg SM, Mulshine JL, Linnoila RI Relationship of p53 overexpression and up-regulation of proliferating cell nuclear antigen with the clinical course of non-small cell lung cancer. Cancer Res 1994;54:2496-503.

19 Dalquen P, Sauter G, Torhorst J, Schultheiss E, Jordan P, Lechmann $S$, et al. Nuclear p53 overexpression is an independent prognostic parameter in node-negative non-small cell lung carcinoma. $\mathcal{F}$ Pathol 1996;178:53-8.

20 Dowell SP, Wilson POG, Derias NW, Lane DP, Hall PA. Clinical utility of the immunocytochemical detection of p53 protein in cytological specimens. Cancer Res 1994;54: p53 protein

21 Clelland CA, Coates M, King-Wai Low D, Ronan J. p53 gene product detection in mesothelioma. Cytopathology [in press].

22 Murakami I, Fujiwara Y, Yamaoka N, Hiyama K, Ishioka S, Yamakido $M$. Detection of p53 gene mutation in cytopathology and biopsy specimens from patients with lung cancer. Am $\mathcal{F}$ Respir Crit Care Med 1996;154:1117-23. 